\title{
Airborne electromagnetic footprints in 1D earths
}

\author{
James E. Reid ${ }^{1}$, Andreas Pfaffling ${ }^{2}$, and Julian Vrbancich ${ }^{3}$
}

\begin{abstract}
Existing estimates of footprint size for airborne electromagnetic (AEM) systems have been based largely on the inductive limit of the response. We present calculations of frequency-domain, AEM-footprint sizes in infinitehorizontal, thin-sheet, and half-space models for the case of finite frequency and conductivity. In a half-space the original definition of the footprint is extended to be the side length of the cube with its top centered below the transmitter that contains the induced currents responsible for $90 \%$ of the secondary field measured at the receiver. For a horizontal, coplanar helicopter frequency-domain system, the in-phase footprint for induction numbers less than 0.4 (thin sheet) or less than 0.6 (half-space) increases from around 3.7 times the flight height at the inductive limit to more than 10 times the flight height. For a vertical-coaxial system the half-space footprint exceeds nine times the flight height for induction numbers less than 0.09. For all models,
\end{abstract}

\section{INTRODUCTION}

A measure of the lateral resolution of an AEM system, the footprint can be used to help assess the applicability of 1D interpretation models and to choose appropriate flightline spacing for surveys (Liu and Becker, 1990) (Kovacs et al., 1995) (Beamish, 2003) (Sattel, 2004). Originally studied in the context of electromagnetic, sea-ice thickness measurements by Liu and Becker (1990), the AEM footprint was defined as the square area centered under the transmitter (Tx) that contained the induced currents responsible for $90 \%$ of the observed secondary magnetic field at the receiver $(\mathrm{Rx})$.

Liu and Becker (1990) calculated the footprint of a closely coupled, frequency-domain, electromagnetic system at the inductive limit and found the footprint size depends on the alti- geometries, and frequencies, the quadrature footprint is approximately half to two-thirds that of the in-phase footprint. These footprint estimates are supported by 3D model calculations that suggest resistive targets must be separated by the footprint dimension for their individual anomalies to be resolved completely.

Analysis of frequency-domain AEM field data acquired for antarctic sea-ice thickness measurements supports the existence of a smaller footprint for the quadrature component in comparison with the in-phase, but the effect is relatively weak. In-phase and quadrature footprints estimated by comparing AEM to drillhole data are considerably smaller than footprints from $1 \mathrm{D}$ and $3 \mathrm{D}$ calculations. However, we consider the footprints estimated directly from field data unreliable since they are based on a drillhole data set that did not adequately define the true, 3D, sea-ice thickness distribution around the AEM flight line. tude of the AEM system $(h)$ as well as on the Tx-Rx geometry. Inductive-limit Liu-Becker footprints for horizontal-coplanar (HCP) and vertical-coaxial (VCX) AEM geometries are 3.73h and $1.35 h$, respectively. An analysis of high-frequency AEM field data by Kovacs et al. (1995) supported the Liu-Becker estimates of footprint size for VCX and HCP geometries. Recently, Reid and Vrbancich (2004) determined inductive-limit footprints for a variety of contemporary AEM systems, including vertical-coplanar (VCP), central-loop, fixed-wing, and towed-bird geometries.

The major limitation of the Liu-Becker footprint is that it is calculated at the inductive limit corresponding to the case of infinite transmitter frequency and/or earth conductivity. Furthermore, at the inductive limit the induced secondary currents are entirely in phase with the primary field of the Tx. The

\footnotetext{
Manuscript received by the Editor August 12, 2004; revised manuscript received May 16, 2005; published online March 10, 2006.

${ }^{1}$ University of Tasmania, School of Earth Sciences, Private Bag 79, Hobart, Tasmania 7001, Australia. E-mail: james.reid@utas.edu.au.

${ }^{2}$ Alfred Wegener Institute for Polar and Marine Research, Division of Climate Physics, P.O. Box 120161, 27515 Bremerhaven, Germany. E-mail: apfaffling@awi-bremerhaven.de.

${ }^{3}$ Defence Science and Technology Organisation, Maritime Operations Division, P.O. Box 44, Pyrmont, New South Wales 2009 , Australia. E-mail: julian.vrbancich@dsto.defence.gov.au.

(C) 2006 Society of Exploration Geophysicists. All rights reserved.
} 
inductive-limit Liu-Becker footprint therefore corresponds to the minimum in-phase footprint of a frequency-domain AEM system. In the case of finite Tx frequency and earth conductivity, the current system induced in the earth will have a greater spatial extent than that at the inductive limit (Beamish, 2003) and it will contain both in-phase and quadrature components.

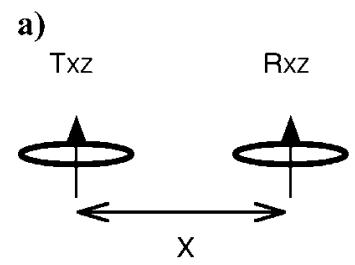

b)

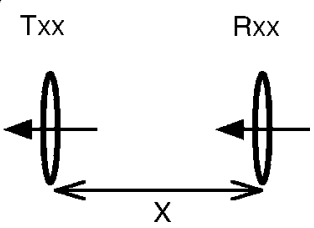

Figure 1. Transmitter-receiver $(\mathrm{Tx}-\mathrm{Rx})$ configurations considered in this study: (a) horizontal coplanar (HCP) (b) vertical coaxial (VCX). In the HCP geometry, Tx and Rx axes are vertical, and the coils are offset in the $x$-direction. In the VCX geometry, the Tx and Rx axes are oriented in the $+x$ direction, and the Tx and $\mathrm{Rx}$ are offset in the $x$-direction. The system flight height above the earth's surface is $h$.

a)
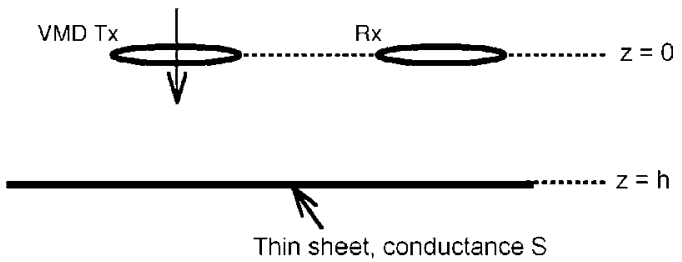

b)

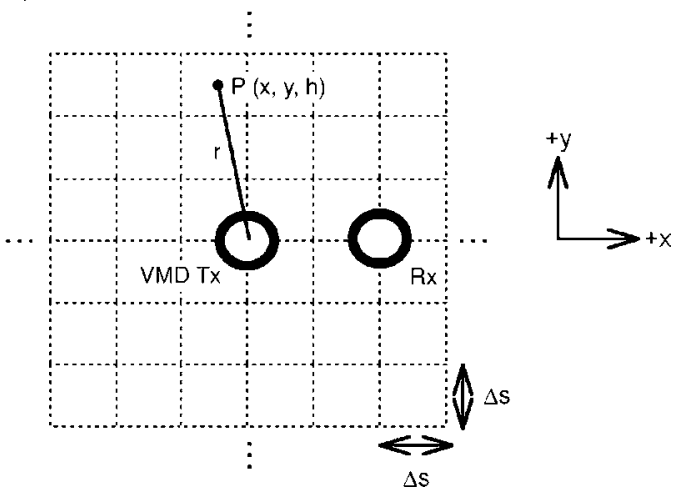

Figure 2. Geometry for calculating the footprint of an HCP AEM system over an infinite horizontal thin sheet: (a) section (b) plan. Panel (b) shows the innermost 36 model cells arranged symmetrically below the Tx position. For practical calculations, discretization of the thin sheet extends much further in the $x$-and $y$-directions than shown on the diagram.
In this paper we extend the Liu-Becker footprint calculation (Liu and Becker, 1990; Reid and Vrbancich, 2004) to the case of finite frequency and earth conductivity. We consider the inphase and quadrature frequency-domain AEM footprints for 1) an HCP AEM system over an infinite, horizontal thin sheet and 2) HCP and VCX geometries over a homogeneous halfspace (Figure 1).

Beamish (2003) has previously calculated the frequencydomain AEM footprints for horizontal and vertical magneticdipole transmitters over a half-space of finite conductivity. His calculations used an alternative definition of the footprint based on the local source-skin distance and were based on the total amplitude of the induced current, rather than on the individual in-phase and quadrature components. Footprints Beamish (2003) calculated were based solely on the current density induced in the half-space by the Tx, whereas the method of Liu and Becker (1990) accounts for the coupling between the induced currents and the receiver Rx.

\section{METHOD}

\section{Infinite thin-sheet model}

The footprint calculation for an infinite, horizontal thin sheet of conductance $S$ is a straightforward extension of the method used to calculate the inductive-limit footprint (Reid and Vrbancich, 2004) because the induced-current system is constrained to lie in the plane of the sheet. In contrast to the inductive-limit calculation, we calculated footprints at a range of frequencies and determined footprint size for both the inphase and quadrature components. The geometry for the thinsheet footprint calculation is illustrated in Figure 2.

The sheet is first discretized into a number of square cells of dimension $\Delta s \times \Delta s$, distributed symmetrically around the transmitter Tx position (Figure 2b). The $x$ - and $y$-components of the total (primary plus secondary) electric field at the chosen frequency are calculated at the center of each cell (see Appendix A, equations A-1 to A-4). The electric field is complex and has both real (in-phase) and imaginary (quadrature) components. The equivalent $x$ - and $y$-directed electric dipole moments $P_{x}$ and $P_{y}$ of each model cell are then given by

$$
P_{x}=\boldsymbol{E}_{x} S(\Delta s)^{2}
$$

and

$$
P_{y}=\boldsymbol{E}_{y} S(\Delta s)^{2} \text {, }
$$

where $E_{x}$ and $E_{y}$ are the (complex) $x$ - and $y$-components of the electric field at the center of each model cell, $S$ is the sheet conductance, and $\Delta s$ is the side length of each cell. The quantity $\mathbf{E} S$ in the above equations equals the surface-current density on the thin sheet $(\mathrm{A} / \mathrm{m})$. Equations 1 and 2 assume that the surface-current density is constant within each cell.

Once equivalent-dipole moments were obtained for all cells, we determined the footprint by integrating the secondary magnetic field contributions at the $\mathrm{Rx}$, from cells within a square area centered beneath the Tx (Liu and Becker, 1990; Reid and Vrbancich, 2004). For the HCP geometry, the required magnetic-field components at $(x, y, z)$ produced by surface currents within a cell centered at $\left(x_{s}, y_{s}, z_{s}\right)$ are

$$
H_{z}^{x d}(x, y, z)=\frac{P_{x}\left(y-y_{s}\right)}{4 \pi r^{3}}
$$


and

$$
H_{z}^{y d}(x, y, z)=-\frac{P_{y}\left(x-x_{s}\right)}{4 \pi r^{3}},
$$

where $\mathbf{H}$ refers to the magnetic field strength and $P_{x}$ and $P_{y}$ are the equivalent $x$ and $y$ electric-dipole moments of the cell and

$$
r=\left[\left(x-x_{s}\right)^{2}+\left(y-y_{s}\right)^{2}+\left(z-z_{s}\right)^{2}\right]^{1 / 2} .
$$

The secondary in-phase magnetic field at the Rx is obtained by substituting the real parts of $P_{x}$ and $P_{y}$ into equations 3 and 4 and into the secondary quadrature magnetic field by using the imaginary parts. The area of integration is increased until the calculated secondary magnetic field is $90 \%$ of the theoretical response at that frequency. The required area of integration is different for the in-phase and quadrature components, as discussed in the Results section that follows. We repeated the process described above for each frequency required.

\section{Homogeneous half-space}

The footprint calculation for a homogeneous half-space is similar to that for an infinite, horizontal thin sheet. However, in a homogeneous half-space the induced in-phase and quadrature current systems diffuse downward and outward from the Tx with decreasing frequency (Reid and Macnae, 1998), whereas in an infinite thin sheet they are constrained to migrate laterally away from the Tx. Therefore, in a half-space we extend the definition of the footprint to be the side length of the cubic volume with its top cantered beneath the Tx that contains the induced currents responsible for $90 \%$ of the secondary field at the Rx at the chosen frequency. This definition was chosen to be consistent with the original 2D footprint defined by Liu and Becker (1990).

The initial calculation step is to discretize a volume of the earth centered beneath the Tx into cubic cells of dimension $\Delta s \times \Delta s \times \Delta s$. At the chosen frequency the complex total electric field resulting from the HMD or VMD Tx then is calculated at the center of each cell (see Appendix A, equations A-5 to A-10). The equivalent $x$ - and $y$-directed electric-dipole moments of each cell are given by

$$
M_{x}=\boldsymbol{E}_{x} \sigma(\Delta s)^{3}
$$

and

$$
M_{y}=\boldsymbol{E}_{y} \sigma(\Delta s)^{3},
$$

where $\mathbf{E}$ is the electric field and $\sigma$ is the half-space conductivity. The quantity $\mathbf{E} \sigma$ in equations 6 and 7 is the current density $\left(\mathrm{A} / \mathrm{m}^{2}\right)$ that is assumed to be constant within each cell.

Once the electric-dipole moments were determined, we calculated the footprint by integrating the secondary magneticfield contributions at the Rx from currents within a cubic volume centered beneath the Tx. We gradually increased the side length of the cube until the integrated response reached $90 \%$ of the theoretical magnetic field at that frequency. For the HCP configuration we require the $z$-components of the magnetic field resulting from $x$ - and $y$-directed dipole moments induced by a VMD Tx. These are given by equations 3 and 4 with equivalent electric-dipole moment quantities $P_{x}$ and $P_{y}$ replaced by $M_{x}$ and $M_{y}$, respectively (equations 6 and 7). For the VCX configuration, with a $+x$-directed HMD source, we also require the $x$-component of the magnetic field due to a $y$-directed electric dipole given by

$$
H_{x}^{y d}(x, y, z)=\frac{M_{y}\left(z-z_{s}\right)}{4 \pi r^{3}} .
$$

In the VCX case the induced $x$-directed, electric-dipole moments do not contribute to the $x$-component of the magnetic field at the Rx.

\section{RESULTS}

As a computational check on the thin-sheet and half-space footprint algorithms described above, the numerical integration of the secondary fields resulting from the induced currents was performed over large areas or volumes, and the resulting, secondary magnetic fields at each frequency were compared with the semianalytic solutions for 1D earths (Kaufman and Keller, 1983, p. 62; Wait, 1982, p. 113). Examples of these calculations are shown in Figure 3. Thin-sheet responses in Figure $3 \mathrm{a}$ were plotted as a function of the dimensionlessinduction number $\alpha=\omega \mu_{0} S h$, where $\omega$ is the angular frequency, $\mu_{0}$ is the magnetic permeability of free space, $S$ is the conductance of the thin sheet, and $h$ is the altitude of the AEM system above the sheet. At all induction numbers, the numerical and analytic thin-sheet solutions agree to within $2 \%$.

VCX half-space responses in Figure $3 b$ were plotted as a function of the induction number $\Theta=\omega \mu_{0} \sigma h^{2}$, where $\sigma$ is the half-space conductivity. The numerical and analytic solutions agree to within $2.3 \%$, except for the quadrature response at the highest induction number, where the error is $\leq 4 \%$. For the HCP geometry (not shown), the numerical and analytic solutions generally agreed to within $1.5 \%$ over the range of induction numbers shown in Figure $3 \mathrm{~b}$ with a maximum error of $2.5 \%$.

Figure 4 shows the HCP in-phase and quadrature footprint sizes for an infinite horizontal thin sheet as a function of the induction number $\alpha$. At high induction numbers, the in-phase footprint approaches the inductive-limit value of $3.73 h$, indicated by the dotted horizontal line. The quadrature footprint approaches a limiting value of $2.10 \mathrm{~h}$. As the induction number decreases, the footprint size for both the in-phase and quadrature components increases markedly because of the outward diffusion of the induced currents from the Tx. The in-phase footprint exceeds $10 h$ for induction numbers $\alpha<0.4$. The quadrature footprint is consistently smaller than the in-phase footprint with the ratio of the two ranging between 1.53 and 2.0 for the selected range of induction numbers (Figure 4).

The HCP and VCX footprints in a homogeneous half-space are shown in Figures 5 and 6, respectively. The in-phase footprints at high induction numbers approach the inductive-limit values of $3.73 h$ (HCP) and $1.35 h$ (VCX). The maximum inphase footprint exceeds $10 h$ for induction numbers $\Theta<0.6$. As for the thin sheet, the quadrature footprint is smaller than the in-phase footprint over the range of induction numbers shown. The ratio of the in-phase to quadrature footprints ranges between 1.55 and 1.72 for the HCP geometry and between 1.54 and 2.01 for VCX.

Our main motivation for conducting this work was to study the lateral resolution of AEM systems. The actual-current system induced in a half-space by a magnetic-dipole transmitter 
Tx has a much greater lateral extent than its depth of penetration into the half-space. Although our definition of the halfspace footprint involves integration over a volume of the subsurface, we found that this definition provides a reasonable measure of the lateral area, giving rise to most of the AEM response at the $\mathrm{Rx}$, as demonstrated by the following examples.

To compare the calculated footprint sizes with the in-phase and quadrature current systems induced in the earth, we consider the case of an HCP system over a homogeneous halfspace. Calculations have been performed for the AWI (Al-

a)

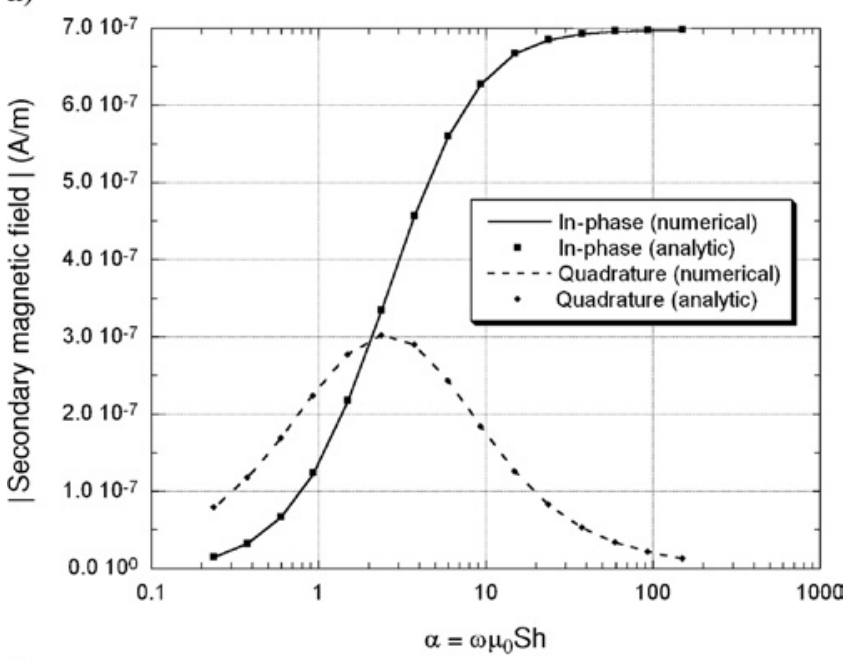

b)

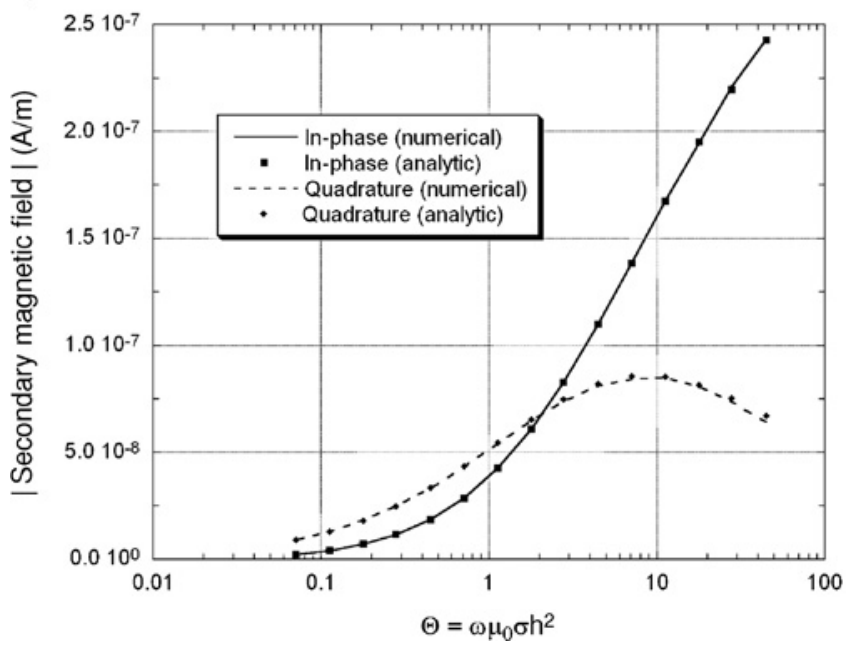

Figure 3. (a) Comparison of the analytic-HCP, secondary magnetic-field response of an infinite horizontal thin sheet to the response obtained by numerical integration of the magnetic-field contributions of the induced in-phase and quadrature surface currents. The thin-sheet conductance is $10 \mathrm{~S}$, Tx-Rx separation is $8 \mathrm{~m}$, and the system flight height above the sheet is $30 \mathrm{~m}$. (b) Comparison of the analytic-VCX, secondary magnetic-field response of a homogeneous halfspace with the response obtained by numerical integration of the magnetic-field contributions of the induced in-phase and quadrature currents. The half-space conductivity is $0.1 \mathrm{~S} / \mathrm{m}$ with the system height and Tx-Rx offset the same as for (a). fred Wegener Institute) HEM-Bird, a modern, fully digital, two-frequency system designed specifically for sea-ice thickness measurements (Pfaffling et al. 2004). The HEM-Bird employs an HCP geometry with a Tx-Rx separation of $2.05 \mathrm{~m}$ at $112 \mathrm{kHz}$ and $2.77 \mathrm{~m}$ at $3680 \mathrm{~Hz}$, although in this example we consider only the lower frequency. The half-space conductivity is $2.77 \mathrm{~S} / \mathrm{m}$, typical of polar seawater, and the system flight height is $15 \mathrm{~m}$. Dimensional in-phase and quadrature footprints for these model parameters are $\approx 69 \mathrm{~m}$ and $\approx 40 \mathrm{~m}$, respectively. A $200-\mathrm{m} \times 200-\mathrm{m} \times 200-\mathrm{m}$ half-space volume was discretized into cubic cells of dimension $2 \mathrm{~m} \times 2 \mathrm{~m} \times 2 \mathrm{~m}$. The magnetic-field contribution of each cell at the $\mathrm{Rx}$ was calculated according to the procedure described in the preceding Method section.

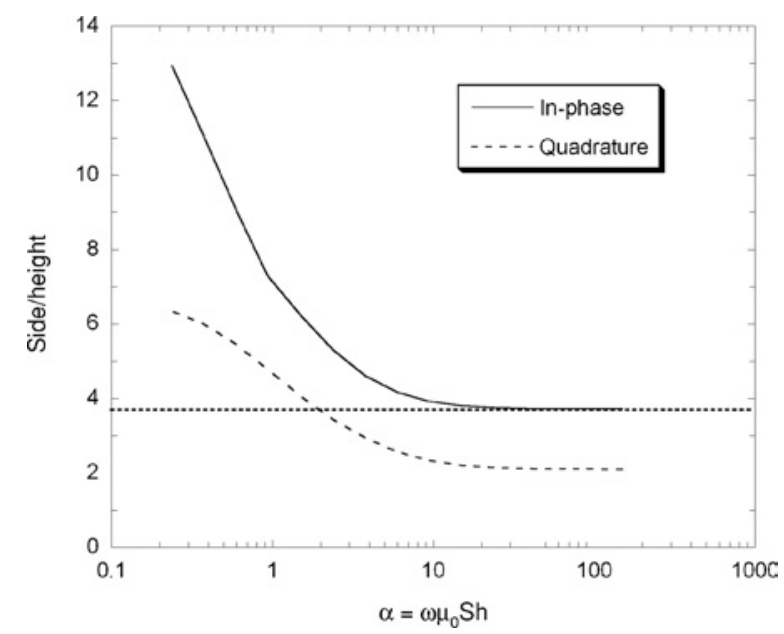

Figure 4. In-phase and quadrature footprints for an HCP AEM system over an infinite, horizontal thin sheet. The footprint is expressed as the side length of the square area of integration normalized by the system height (side/height). The lateral Tx-Rx separation is $8 \mathrm{~m}$. The horizontal dotted line indicates the inductive-limit, in-phase footprint.

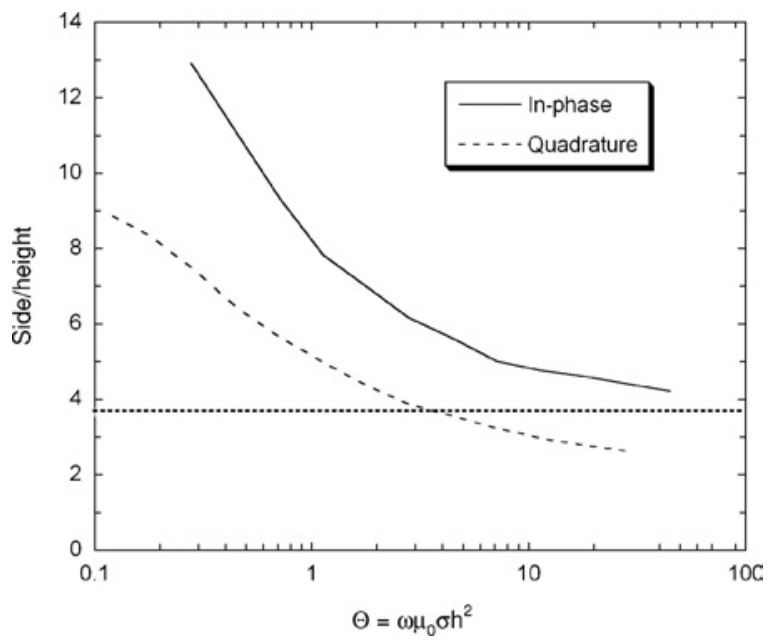

Figure 5. In-phase and quadrature footprints for an HCP AEM system over a homogeneous half-space. The lateral Tx$\mathrm{Rx}$ separation is $8 \mathrm{~m}$. The horizontal dotted line indicates the inductive-limit, in-phase footprint. 
Figure 7 shows a horizontal slice through the half-space at a depth of $3 \mathrm{~m}$ below the surface, representing a layer of model cells extending from $2 \mathrm{~m}$ to $4 \mathrm{~m}$ depth. The magnetic-field contributions of each cell at the $\mathrm{Rx}$ are shown as a grayscale image with darker colors indicating stronger contributions. The more-compact pattern of the quadrature contributions to the magnetic field is immediately apparent and supports the smaller footprint for this component. The lateral extents of the calculated footprints for the in-phase and quadrature components are indicated by heavy black squares in Figures $7 \mathrm{a}$ and $7 \mathrm{~b}$. Although calculated by integration over a volume of the subsurface, the footprint provides a reasonable estimate of the lateral extent of the region making the strongest contributions to the magnetic field.

Figure 8 shows a cross section through the half-space in the plane $y=1 \mathrm{~m}$, representing model cells extending from $y=$ $0 \mathrm{~m}$ to $y=2 \mathrm{~m}$. The heavy vertical lines on Figures $8 \mathrm{a}$ and $8 \mathrm{~b}$ indicate the lateral extents of the in-phase and quadrature footprints. In both cases the volume of integration used for the footprint calculation extends beneath the maximum depth of the cross section shown.

\section{THREE-DIMENSIONAL MODELING}

To further test our estimates of the HEM footprint, we calculated a series of 3D EM models using the code MARCO_AIR version 2.6.2 (Xiong, 1992; Xiong and Tripp, 1995; Raiche, 2001; Vrbancich et al., 2004). Calculations were performed for the AWI HEM-Bird sea-ice thickness system at a frequency of $3680 \mathrm{~Hz}$. The 3D model geometry is illustrated in Figure 9e. The model consists of two identical 0.05$\mathrm{S} / \mathrm{m}$ block targets located in a two-layered host medium. The 0.5 -m-thick upper layer of the host medium represents sea ice (conductivity $0.05 \mathrm{~S} / \mathrm{m}$ ), and the lower layer represents seawater (conductivity $2.77 \mathrm{~S} / \mathrm{m}$ ). The resistive-block targets represent zones of thicker sea ice (pressure ridges) with strike extent $50 \mathrm{~m}$, in-line extent (width) $15 \mathrm{~m}$, and depth extent $2 \mathrm{~m}$.

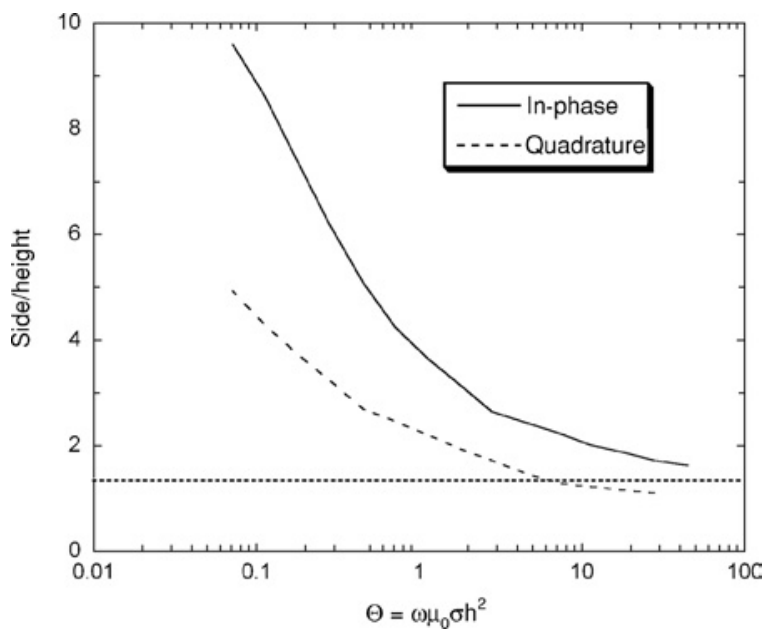

Figure 6. In-phase and quadrature footprints for a VCX AEM system over a homogeneous half-space. The lateral Tx-Rx separation is $8 \mathrm{~m}$. The horizontal dotted line indicates the inductive-limit in-phase footprint.
For this model we used MARCO_AIR to compute responses for a range of target separations. In-phase and quadrature responses for two separations are shown in Figure 9, with the response of the two-layered host medium also plotted for comparison. Figures $9 \mathrm{a}$ and $9 \mathrm{~b}$ show the $3680 \mathrm{-Hz}$ in-phase and quadrature responses for a target separation of $45 \mathrm{~m}$. For a flight height of $15.5 \mathrm{~m}$ above the seawater, this target separation is slightly larger than the theoretical quadrature footprint. At the center of the profile, the quadrature response of the $3 \mathrm{D}$ model is equal to the response of the two-layered host medium, whereas the in-phase response is not. This indicates that the AEM anomalies resulting from the individual targets have been resolved just in the quadrature component but not in the in-phase.

Figures 9c and 9d show the responses calculated for an increased target separation of $75 \mathrm{~m}$, slightly larger than the

a)

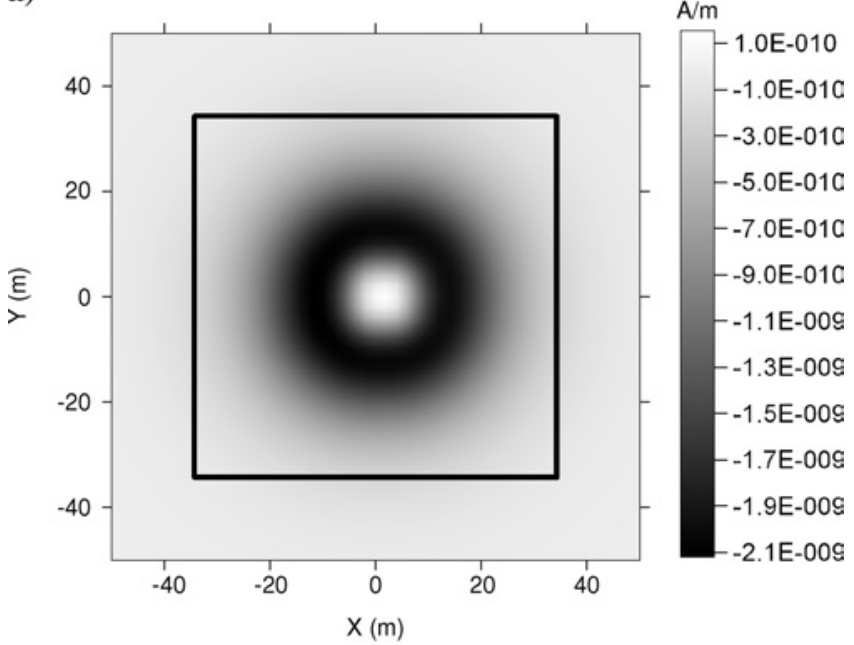

b)

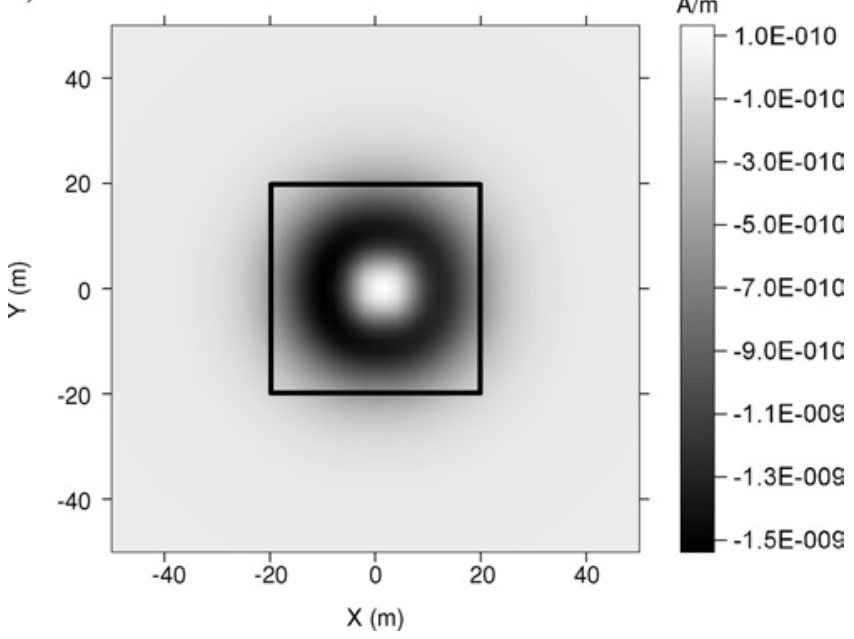

Figure 7. HCP in-phase (a) and quadrature (b) magnetic-field contributions from induced currents at a depth of $3 \mathrm{~m}$ below the surface of a $2.77-\mathrm{S} / \mathrm{m}$ homogeneous half-space. The airborne Tx and Rx are located at $(x, y, z)=(0 \mathrm{~m}, 0 \mathrm{~m}, 15 \mathrm{~m})$ and $(2.77 \mathrm{~m}, 0 \mathrm{~m}, 15 \mathrm{~m})$, respectively. The Tx frequency is $3680 \mathrm{~Hz}$. The bold square on each panel indicates the lateral extent of the AEM footprint as calculated in the main text. 


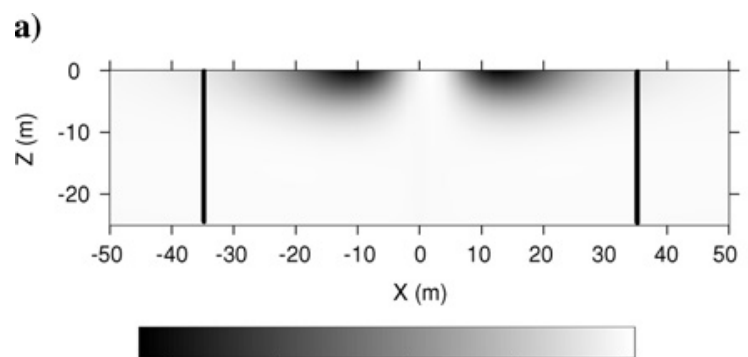

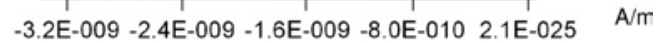

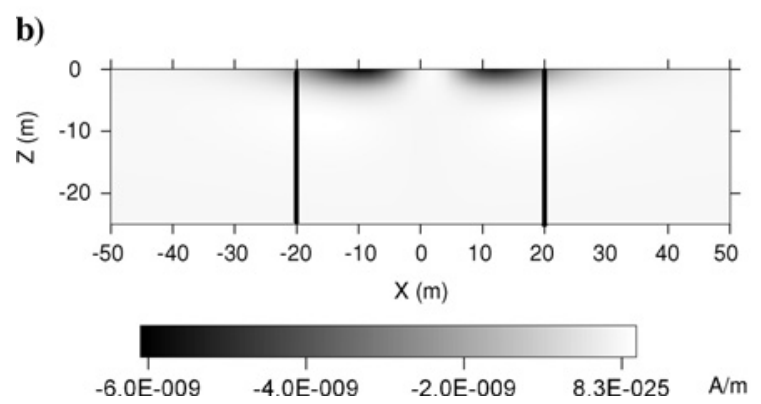

Figure 8. HCP in-phase (a) and quadrature (b) magnetic-field contributions from induced currents in the plane $y=1 \mathrm{~m}$, for the model parameters described in the caption to Figure 7. The bold vertical lines on each panel denote the lateral extent of the AEM footprint as calculated in the main text.

a)

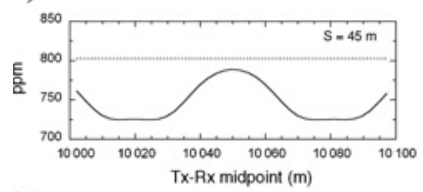

b)

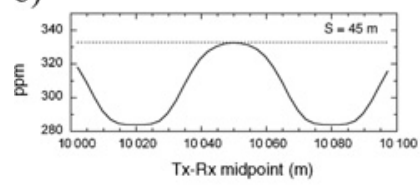

e)

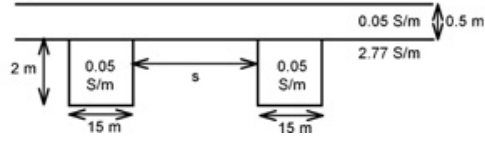

Figure 9. MARCO_AIR 3D EM model results for a sea-ice model containing two identical block targets embedded in a two-layered host medium. Responses were computed for the HCP AWI HEM-Bird system with frequency $3680 \mathrm{~Hz}$ and Tx$\mathrm{Rx}$ separation of $2.77 \mathrm{~m}$. Flight height was $15 \mathrm{~m}$ above the surface. The profile crosses directly over the center of the targets that have strike extent $50 \mathrm{~m}$ and in-line extent (width) $15 \mathrm{~m}$. Panels (a) and (b) show in-phase and quadrature responses for a target separation of $45 \mathrm{~m}$ (target centers at Tx-Rx midpoints of $10020 \mathrm{~m}$ and $10080 \mathrm{~m}$ ). Panels (c) and (d) show the corresponding responses for a target separation of $75 \mathrm{~m}$ (target centers at Tx-Rx midpoints of $10005 \mathrm{~m}$ and $10095 \mathrm{~m}$ ). The horizontal dotted lines in each panel show the response of the two-layered host $(0.5 \mathrm{~m}$ thick sea ice overlying seawater) without the targets present. The model geometry is shown in (e). theoretical in-phase footprint. In this case, both the in-phase and quadrature responses at the center of the profile are equal to the host response. The individual target anomalies are just resolved in the in-phase profile but have been completely resolved in the quadrature profile. It should be noted that because the targets are only $15-\mathrm{m}$ wide, the model response at the center of each target does not reach the $1 \mathrm{D}$ response expected for a 2.5-m-thick layer of sea ice overlying seawater that is $609 \mathrm{ppm}$ and $223 \mathrm{ppm}$ for the in-phase and quadrature components, respectively.

Thus, the 3D models support the calculated half-space footprint sizes for the AWI HEM system. The model results indicate that the resistive targets must be separated by roughly the footprint size if their anomalies are to be resolved completely.

\section{FIELD EXAMPLE}

The theoretical calculations above suggest a smaller footprint for the quadrature component of the frequency-domain response in comparison with the in-phase response. To test whether this effect can be observed in real data, we consider a helicopter electromagnetic (HEM) data set that was acquired for sea-ice thickness measurements in the antarctic and that was verified by extensive drilling. AEM measurements of seaice thickness have been described by Kovacs and Holladay (1990) and Kovacs et al. (1995).

To analyze the AEM field data for footprint size, the EM and drillhole data sets were converted from sea-ice thickness to draft, i.e., depth extent of sea ice below sea level (Figure 10). This step is required because the sea-ice thickness is calculated from AEM data, based on (1) the depth to seawater interpreted from the EM data and (2) the altitude of the AEM system above the upper sea-ice surface measured by a laser altimeter. At pressure ridges, deformed sea ice typically has an above-surface topographic expression referred to as the ridge sail, as well as a subsurface extent or keel (Figure 10). The small pressure ridge sails produce little or no measurable effect on the AEM response but can be detected easily by the laser altimeter that has a height resolution and lateral footprint of only a few centimeters. In these cases the sea-ice thickness interpreted from the AEM data contains thickness anomalies of high spatial frequency solely because of the above-surface topography at ridges. This problem com-

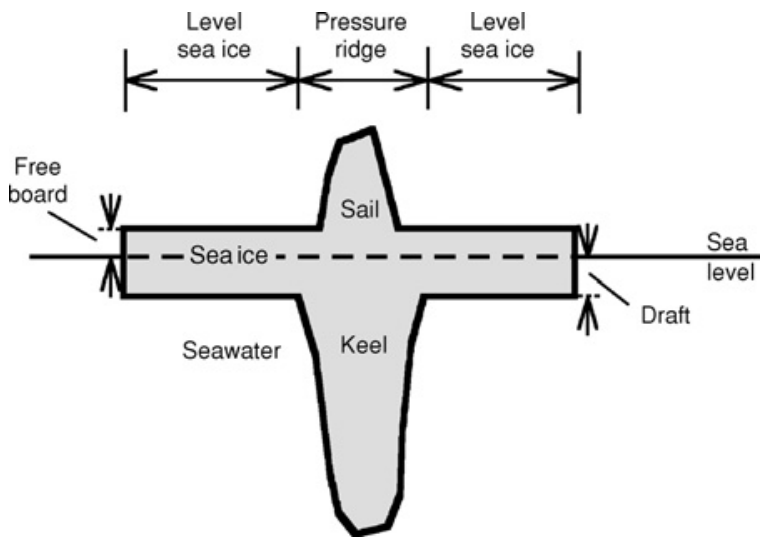

Figure 10. Schematic cross section through a sea-ice floe that illustrates quantities referred to in the main text. Diagram is not to scale. 
plicates EM-footprint determination from field data. However, based on measurements of depth to seawater routinely made during drilling, conversion of EM-determined thicknesses to draft removes the anomalies solely because of the laser altimeter.

Figure 11a shows profiles of sea-ice draft from three parallel transects across an antarctic sea-ice floe. The profiles cross areas of level sea ice of relatively constant thickness and also zones of thick, deformed sea ice (pressure ridges) where the drilled draft is highly variable. For each 500-m-long profile, drilled measurements were conducted approximately every $2 \mathrm{~m}$ in areas of level sea ice and every $1 \mathrm{~m}$ close to pressure ridges, resulting in a total of 976 holes. The lateral separation between adjacent profiles was $20 \mathrm{~m}$.

Large variations in sea-ice draft are apparent along each profile and also between adjacent profiles, indicating a strongly 3D sea-ice thickness distribution. Mean and modal sea-ice drafts on all three drilled profiles are $1.08 \mathrm{~m}$ and $0.48 \mathrm{~m}$, respectively. Additional drillhole measurements between the survey lines were originally planned in order to better characterize the $3 \mathrm{D}$ thickness distribution but were not completed

a)

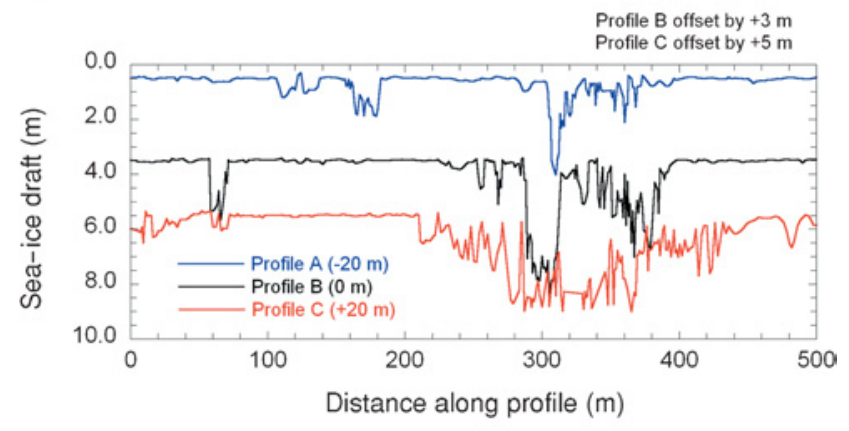

b)

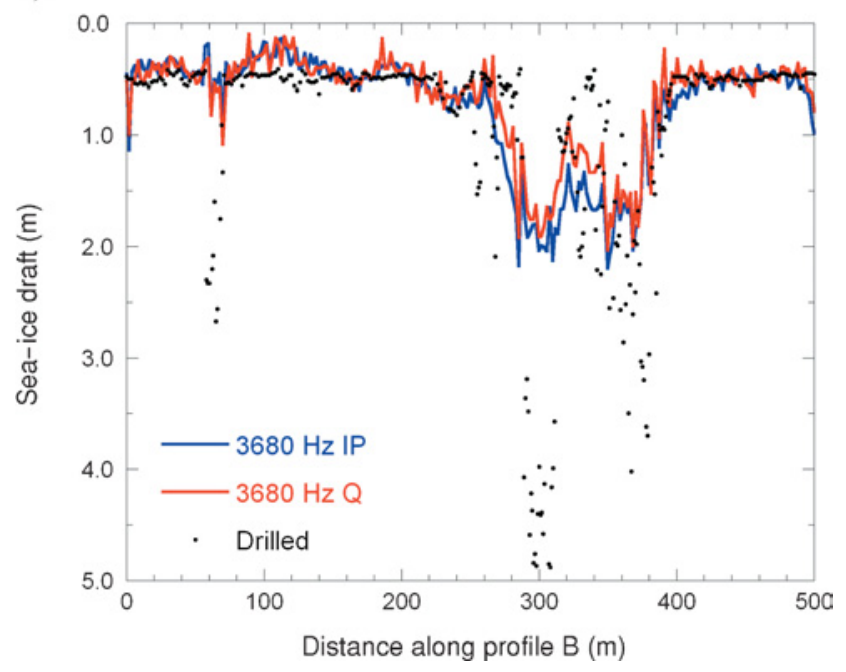

Figure 11. (a) Drilled sea-ice draft along three parallel 500$m$-long profiles in antarctic sea ice. The spacing between profiles is $20 \mathrm{~m}$. For clarity, Profiles B and C have been offset by $+3 \mathrm{~m}$ and $+5 \mathrm{~m}$, respectively. (b) Comparison of the drilled sea-ice draft on the center Profile B to draft estimated by independent, empirical interpretation of the $3680 \mathrm{~Hz}$ in-phase and $3680 \mathrm{~Hz}$ quadrature HEM data. because of time constraints in the field. Although the thickness distribution was adequately sampled in the in-line direction (drillhole spacing 1-2 m), it was highly undersampled in the direction perpendicular to the profiles (sample spacing $20 \mathrm{~m})$.

We used the AWI HEM-Bird system to collect AEM data on the center profile (Profile B). The height of the AEM system was measured with a laser altimeter mounted in the bird. Mean flight height above the snow surface was $14.6 \mathrm{~m}$. The HEM data were processed to remove the effects of drift (Valleau, 2000), but no other filtering was applied. Our field-data analysis considers only $3680 \mathrm{~Hz}$ data because we anticipated that the in-phase footprint size at this frequency would show the greatest difference from the established inductive-limit value $(3.7 \mathrm{~h})$. The $112-\mathrm{kHz}$ data were not used because of an electronic problem that resulted in severe noise contamination of the quadrature response.

Noise levels for the $3680 \mathrm{~Hz}$ response were estimated directly from field data recorded over open water and were based on the residuals between the observed data and the predicted theoretical response of a $2.77-\mathrm{S} / \mathrm{m}$ half-space at a range of flight heights. Standard deviations for the in-phase and quadrature data were 6.4 and $5.8 \mathrm{ppm}$, respectively. Seaice thickness was interpreted separately from the $3680 \mathrm{~Hz}$ inphase and quadrature responses using an empirical technique routinely employed at AWI for AEM sea-ice thickness interpretation (Pfaffling et al., 2004). The amplitude of the measured AEM data shows an exponential dependence on height above seawater. A good fit to the measured EM response as a function of height can be obtained using a function of the form

$$
Z=C_{1}+C_{2} \cdot e^{C_{3} \cdot h}
$$

where $Z$ denotes the response amplitude and $h$ the height of the system above the seawater. For practical interpretation, best-fit values for the coefficients $C_{1}, C_{2}$, and $C_{3}$ are determined from AEM data recorded over areas of open (ice-free) water several times during each survey flight. In areas of seaice cover, equation 9 can be inverted to estimate the height of the AEM system above the sea-ice seawater interface. Snow plus sea-ice thickness is calculated by subtracting the bird altitude from the estimated depth to seawater. This extremely rapid empirical approach can be applied to individual in-phase or quadrature components of the measured response. However, it has the disadvantage of not accounting for the effect of the finite conductivity of the sea ice.

Figure 11b shows sea-ice draft interpreted from $3680 \mathrm{~Hz}$ in-phase and quadrature data acquired on Profile B. We calculated draft by subtracting the sea-ice freeboard (i.e., ice thickness above sea level) from the AEM-determined, seaice thickness at each point on the profile. We estimated the freeboard at each AEM measurement point using spline interpolation between drilled measurements. Both in-phase and quadrature components of the AEM data give very good estimates of the draft over level ice; e.g., between $400 \mathrm{~m}$ and $500 \mathrm{~m}$ and at most points on the profile, the draft derived from both components shows very close agreement.

The sea-ice draft at major pressure ridges is underestimated because the EM response yields an average depth to seawater over the footprint (Kovacs et al., 1995). The maximum- 
interpreted draft at the two major pressure ridges is very similar for both the in-phase and quadrature profiles. However, the quadrature component appears to show better lateral resolution of the two prominent ridges at $300 \mathrm{~m}$ and $370 \mathrm{~m}$ compared to the in-phase data and gives a better indication of the thin sea ice between the ridges at $340 \mathrm{~m}$. This supports the existence of a smaller footprint for the quadrature component in comparison with the in-phase - in agreement with the theory. The minor pressure ridge at $70 \mathrm{~m}$ produces no detectable anomaly in either the $3680-\mathrm{Hz}$ in-phase or quadrature components. Aerial photographs taken during HEM data acquisition indicate that this ridge is a local feature with a strike extent of approximately $20 \mathrm{~m}$ and an in-line extent (width) of around $13 \mathrm{~m}$ (Figure 11a). Because of the small size of the feature in comparison with the EM footprint, it cannot be detected by AEM measurements.

In an attempt to estimate the AWI HEM-Bird footprint directly from the field data, we compared the sea-ice draft estimated from the $3680 \mathrm{~Hz}$ in-phase and quadrature responses to a moving average of the drilled draft within square windows of varying size. For each window size, we have calculated an rms error of fit between the HEM-determined and drilled profiles given by

$$
\operatorname{RMS}(\%)=100 \sqrt{\frac{\sum_{i=1}^{N}\left(\frac{z_{i}^{w}-z_{i}^{E M}}{z_{i}^{w}}\right)^{2}}{N}},
$$

where $N$ is the number of points on the profile, $z_{i}^{E M}$ is the draft determined from the (in-phase or quadrature) HEM response at the $i$ th point, and $z_{i}^{w}$ is the weighted average drillhole draft within a square window centered at the $i$ th point.

Because most of the contribution to the response at the receiver Rx comes from an annular region roughly coincident with the total electric-field maximum in the half-space (Figures 7 and 8), we have weighted the drillhole measurements within the footprint depending on their distance from the TxRx midpoint of the HEM system, rather than simply calculating the mean sea-ice draft within the footprint. The weights are the magnetic-field contributions at the $\mathrm{Rx}$ from the surface of a half-space and were calculated at $3680 \mathrm{~Hz}$ for a system at height $15.12 \mathrm{~m}$ above a $2.765-\mathrm{S} / \mathrm{m}$ half-space. The chosen height is equal to the mean flight height above the surface plus the modal thickness of the snow plus sea ice from all three profiles. The in-phase and quadrature weighting functions are shown in Figure 12a.

The smallest rms error of fit between the averaged drillhole measurements and the EM-determined draft occured for footprint sizes of $11 \mathrm{~m}(0.75 h)$ for the quadrature component and $36 \mathrm{~m}(2.4 \mathrm{~h})$ for the in-phase. Figure $12 \mathrm{~b}$ shows a comparison of the sea-ice draft determined from the $3680-\mathrm{Hz}$ in-phase data with the averaged drillhole measurements over windows of $69 \mathrm{~m}$, corresponding to the theoretical footprint, and $36 \mathrm{~m}$, determined from direct comparison of EM and drillhole data described above. Figure 12c shows a corresponding plot for the $3680-\mathrm{Hz}$ quadrature component with a theoretical footprint of $41 \mathrm{~m}$ and a drillhole-determined footprint of $11 \mathrm{~m}$.

For both the in-phase and quadrature components, averaging over the theoretical footprint gives a reasonable estimate of the maximum EM-determined sea-ice draft at major pressure ridges but tends to oversmooth the lateral variations in the estimated draft along the profile. Overestimation of the level ice draft between $400 \mathrm{~m}$ and $500 \mathrm{~m}$ occurs for both the in-phase and quadrature theoretical footprints since these include contributions from the thick sea ice $(\approx 2 \mathrm{~m})$ drilled between $400 \mathrm{~m}$ and $500 \mathrm{~m}$ on Figure 12c.

The smaller footprints that were determined by comparison of drillhole to EM-determined sea-ice drafts strongly overes-

a)

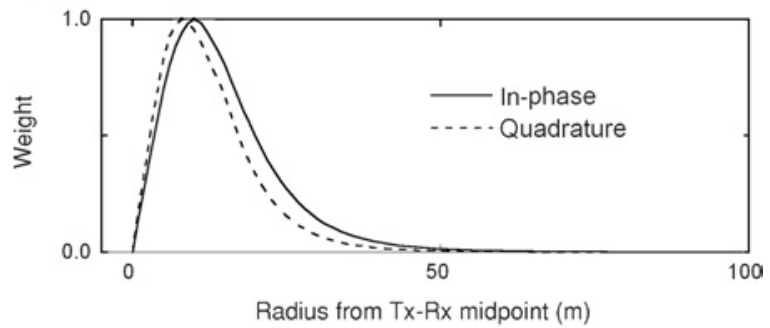

b)

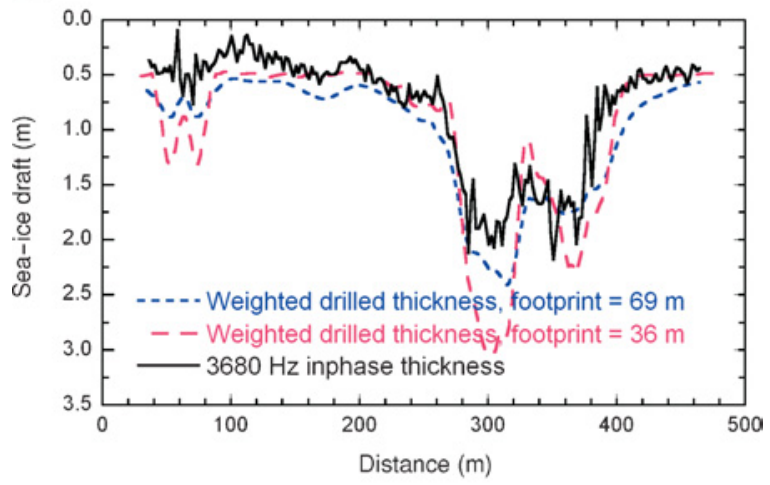

c)

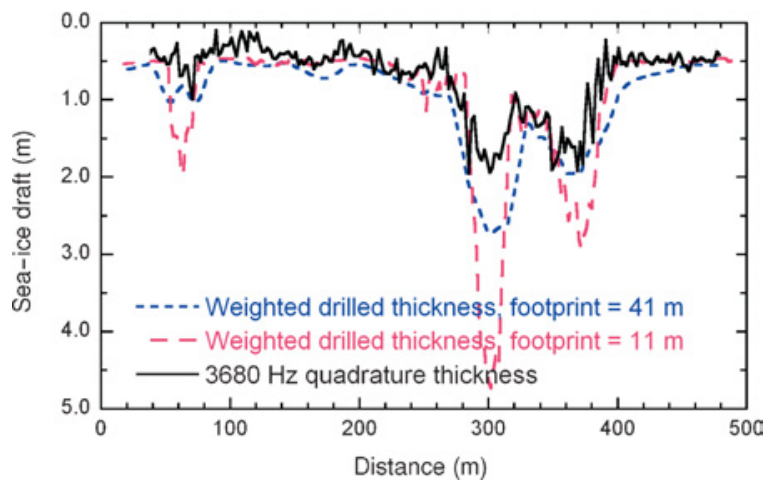

Figure 12. (a) $3680 \mathrm{~Hz}$ in-phase and quadrature weighting functions. Calculation of these weights is described in the main text. (b) Comparison of the $3680 \mathrm{~Hz}$ in-phase sea-ice draft (solid line) with moving weighted averages of drillhole data within square windows of dimension $36 \mathrm{~m}$ and $69 \mathrm{~m}$. The $69 \mathrm{~m}$ window corresponds to the theoretical $3680 \mathrm{~Hz}$ inphase footprint and the $36 \mathrm{~m}$ window to the footprint that gives the smallest rms error of fit between the drilled and EM-determined sea-ice draft. (c) Comparison of the $3680 \mathrm{~Hz}$ quadrature sea-ice draft (solid line) to moving weighted averages of drillhole data within square windows of dimensions $41-\mathrm{m}$ and $11 \mathrm{~m}$. The $41-\mathrm{m}$ window corresponds to the theoretical $3680-\mathrm{Hz}$ quadrature footprint and the $11-\mathrm{m}$ window to the footprint that gives the smallest rms error of fit between the drilled and EM-determined sea-ice draft. 
timate the maximum EM draft at pressure ridges but give a more reliable representation of lateral variation in the estimated draft and level sea-ice thickness.

\section{DISCUSSION}

The difference between the footprints estimated from the field data and the theoretical values probably arises because the drillhole measurements did not completely characterize the sea-ice thickness distribution at the field site. The drillhole field data and aerial photographs taken during AEM data acquisition indicate that the actual sea-ice thickness distribution is strongly $3 \mathrm{D}$. Tests using synthetic $3 \mathrm{D}$ drillhole data sets have shown that the weighted averages of the drilled thicknesses over the footprint can be biased strongly when the actual thickness distribution is undersampled in the strike direction (perpendicular to the profiles), as was the case for our field experiment. Undersampling of the drilled thickness distribution in the strike direction can result in the effects seen in Figures 11b and 11c, such as oversmoothing of lateral variations along the profiles and significant overestimation of the thickness in level sea-ice areas. Accordingly, we consider that the small footprints estimated from direct comparison of the AEM-determined and drilled drafts are likely to be underestimates of the actual footprint - especially since they considerably overestimate the EM-determined sea-ice draft at the major pressure ridges.

Kovacs et al. (1995) used autocorrelation analysis to determine the footprint of a HEM system for sea-ice thickness measurements. They were able to achieve good agreement between the footprint estimated from high-frequency field data and the inductive-limit, footprint estimates of Liu and Becker (1990). We performed a 1D autocorrelation analysis of the $3680-\mathrm{Hz}$ HEM response using drillhole data from Profile B only, following the approach described by Kovacs et al. (1995). For the purpose of the autocorrelation calculation, the drillhole data were resampled at a uniform spacing of $2 \mathrm{~m}$. This analysis yielded footprint estimates of $91 \mathrm{~m}(5.72 \mathrm{~h})$ and $71 \mathrm{~m}(4.47 \mathrm{~h})$, respectively, for the $3680 \mathrm{~Hz}$ in-phase and quadrature components. Although the indicated quadrature footprint is again smaller than the in-phase footprint, the autocorrelation estimates considerably exceed the theoretical footprints.

The reasons for this poor agreement have not been established but are most probably related to the strongly $3 \mathrm{D}$ seaice thickness distribution at our antarctic field site. We also note that Kovacs et al. (1995) performed their autocorrelation analysis on HEM sea-ice thickness estimated using simultaneous $1 \mathrm{D}$ inversion of both the in-phase and quadrature components of the response, whereas we used HEM sea-ice drafts derived independently from the in-phase and quadrature data by empirical means.

\section{CONCLUSIONS}

Theoretical footprint calculations in infinite thin-sheet and half-space models have shown that for finite frequencies and conductivities, frequency-domain AEM footprint sizes may be several times the widely accepted, inductive-limit values. In both half-space and thin-sheet models, the theoretical quadrature footprint is considerably smaller than the in-phase footprint at all frequencies. For the calculations presented in this paper, the ratio of the theoretical HCP in-phase to quadrature footprint sizes for a horizontal thin-sheet model is between 1.53 and 2.0. For the half-space model, the ratio is between 1.55 and 1.72 for the HCP geometry, and between 1.54 and 2.01 for VCX.

The theoretical footprint calculations presented in Figures 4-6 are strictly valid only for frequency-domain systems with Tx-Rx separation of $8 \mathrm{~m}$. However, the theoretical analysis can be extended easily to other frequency-domain geometries and to the time domain.

The in-phase and quadrature footprint estimates based on 1D models have been supported by previous analysis of HEM field data (Kovacs et al., 1995) and also by 3D modeling conducted during this study. Our 3D MARCO_AIR model results also suggest a smaller footprint for the quadrature component in comparison with the in-phase, as well as indicate that seaice pressure ridges must be separated by roughly one footprint for their anomalies to be resolved completely. Our attempt to determine the in-phase and quadrature footprints directly from the field data resulted in estimated footprints that are considerably smaller than the theoretical values. We believe that our field estimates of footprint size are unreliable because the drillhole data did not define adequately the true $3 \mathrm{D}$ seaice thickness distribution perpendicular to the survey line. Reliable estimation of the practical footprint from field data will require more detailed $2 \mathrm{D}$ drilling around the AEM flight line. A field experiment of this nature is planned for an upcoming HEM survey in the Arctic.

\section{ACKNOWLEDGMENTS}

This research was supported in part by Australian Antarctic Science Advisory Committee Grant 2381. The field data shown in Figures 11 and 12 were acquired in September 2003 as part of the Antarctic Remote Ice Sensing Experiment (ARISE). We thank Tony Worby, John Bishop, Kazutaka Tateyama, Angus Munro, and many others for performing almost 1000 drillhole measurements of sea-ice thickness, as well as the officers and crew of the RSV Aurora Australis for field support. Comments from three anonymous reviewers and the associate and assistant editors greatly improved the original manuscript.

\section{APPENDIX A}

\section{ELECTRIC FIELDS INDUCED BY AIRBORNE MAGNETIC-DIPOLE TRANSMITTERS OVER INFINITE HORIZONTAL THIN-SHEET AND HOMOGENEOUS HALF-SPACE MODELS}

\author{
Infinite, horizontal thin-sheet model $-+z$-directed \\ VMD source
}

The quasi-static, total electric field on the surface of an infinite thin sheet of conductance $S$, because of a vertical magnetic-dipole (VMD) Tx at height $h$ above the sheet, can be obtained from vector potentials given by Kaufman and Keller (1983, p. 62). In cylindrical coordinates with the origin at the VMD Tx, the azimuthal electric field at the thin sheet 
$(z=h)$ is

$$
E_{\phi}^{t}(r, h)=-\frac{i \omega \mu_{0} M}{2 \pi} \int_{0}^{\infty} \frac{\lambda^{2}}{\left(2 \lambda-i \omega \mu_{0} S\right)} e^{-\lambda h} J_{1}(\lambda r) d \lambda,
$$

where

$$
r=\sqrt{x^{2}+y^{2}} .
$$

$\omega$ is the angular frequency (radians/sec), $\mu_{0}$ is the magnetic permeability of free space (henry/m), $M$ is the Tx moment $\left(\mathrm{Am}^{2}\right)$, and $\lambda$ is the wavenumber.

The Cartesian components of the electric field are then given by

$$
E_{x}^{t}=-\frac{y}{r} E_{\phi}^{t}
$$

and

$$
E_{y}^{t}=\frac{x}{r} E_{\phi}^{t}
$$

\section{Homogeneous half-space}

The quasi-static, total electric field within a half-space of conductivity $\sigma$, because of an airborne VMD or HMD Tx can be obtained from equations for the vector potential (Ward, 1967). In the following equations the Tx is located at height $h$ above the surface of the half-space.

\section{$+z$-directed VMD source}

In cylindrical coordinates with the origin on the surface of the half-space directly beneath the Tx, the azimuthal component of the total electric field within the earth $(z \geq 0)$ is given by

$$
\begin{aligned}
E_{\phi}^{t}= & \frac{i \omega \mu_{0} M}{2 \pi} \int_{0}^{\infty} \frac{\lambda^{2}}{\left(\lambda+u_{1}\right)} \\
& \times e^{\left(u_{1}-\lambda\right) h} e^{-u_{1} z} J_{1}(\lambda r) d \lambda, \quad(z \geq 0),
\end{aligned}
$$

where

$$
u_{1}=\sqrt{\lambda^{2}-i \omega \mu_{0} \sigma} .
$$

The Cartesian components of the electric field are given by equations A-3 and A-4.

\section{$+x$-directed HMD source}

The $x$ and $y$-components of the total electric field within the half-space $(z \geq 0)$ are given by

$$
E_{x}^{t}=-\frac{i \omega \mu_{0} M}{2 \pi}\left[\frac{2 x y}{r^{3}} I_{1}-\frac{x y}{r^{2}} I_{2}\right]
$$

and

$$
E_{y}^{t}=-\frac{i \omega \mu_{0} M}{2 \pi}\left[\frac{x_{2}}{r_{2}} I_{2}-\frac{\left(x^{2}-y^{2}\right)}{r^{3}} I_{1}\right],
$$

where

$$
I_{1}=\int_{0}^{\infty} \frac{\lambda}{\left(\lambda+u_{1}\right)} e^{\left(u_{1}-\lambda\right) h} e^{-u_{1} z} J_{1}(\lambda r) d \lambda \quad(z \geq 0)
$$

and

$$
I_{2}=\int_{0}^{\infty} \frac{\lambda^{2}}{\left(\lambda+u_{1}\right)} e^{\left(u_{1}-\lambda\right) h} e^{-u_{1} z} J_{0}(\lambda r) d \lambda \quad(z \geq 0) .
$$

\section{REFERENCES}

Beamish, D., 2003, Airborne EM footprints: Geophysical Prospecting, 51, 49-60.

Kaufman, A. A., and G. V. Keller, 1983, Frequency and transient soundings: Elsevier Science Publishing Company, Inc.

Kovacs, A., and J. S. Holladay, 1990, Sea ice thickness measurement using a small airborne electromagnetic sounding system: Geophysics, 55, 1327-1337.

Kovacs, A., J. S. Holladay, and C. J. J. Bergeron, 1995, The footprint/altitude ratio for helicopter electromagnetic sounding of sea ice thickness: comparison of theoretical and field estimates: Geophysics, 60, 374-380.

Liu, G., and A. Becker, 1990, Two-dimensional mapping of sea ice keels with airborne electromagnetics: Geophysics, 55, 239-248.

Pfaffling, A., C. Haas, and J. E. Reid, 2004, Empirical processing of HEM data for sea ice thickness mapping: 10th European Meeting of Environmental and Engineering Geophysics, Extended Abstracts.

Raiche, A., 2001, Choosing an AEM system to look for kimberlites a modeling study: Exploration Geophysics, 32,1-8.

Reid, J. E., and J. C. Macnae, 1998, Comments on the electromagnetic 'smoke ring' concept: Geophysics, 63, 1908-1913.

Reid, J. E., and J. Vrbancich, 2004, A comparison of the inductivelimit footprints of airborne electromagnetic configurations: Geophysics, 69, 1229-1239.

Sattel, D., 2004, The resolution of shallow horizontal structure with airborne EM: Exploration Geophysics, 35, 208-216.

Valleau, N. C., 2000, HEM data processing - a practical overview: Exploration Geophysics, 31, 584-594.

Vrbancich, J., J. E. Reid, A. Pfaffling, D. W. Annetts, and A. P. Worby, 2004, 3D EM modeling of Antarctic sea ice pressure ridges: 17th Geophysical Conference, Australian Society of Exploration Geophysicists, Extended Abstracts.

Wait, J. R., 1982, Geo-electromagnetism: Academic Press Inc.

Ward, S. H., 1967, Electromagnetic theory for geophysical applications, in Hansen, D. A., W. E. Heinrichs, R. C. Holmer, R. E. MacDougall, G. R. Rogers, J. S. Sumner, and S. H. Ward (Eds.), 1967, Mining geophysics, volume 2, Theory: SEG, 10-196.

Xiong, Z., 1992, Electromagnetic modeling of 3-D structures by the method of system iteration using integral equations: Geophysics, 57, 1556-1561.

Xiong, Z., and A. C. Tripp, 1995, A block iterative algorithm for 3 -D electromagnetic modeling using integral equations with symmetized substructures: Geophysics, 60, 291-295. 